\title{
EVOLUTION AND BREAKUP OF VISCOUS ROTATING DROPS*
}

\author{
M. A. FONTELOS ${ }^{\dagger}$, V. J. GARCÍA-GARRIDO ${ }^{\dagger}$, AND U. KINDELÁN ${ }^{\ddagger}$
}

\begin{abstract}
We study the evolution of a viscous fluid drop rotating about a fixed axis at constant angular velocity $\Omega$ or constant angular momentum $L$ surrounded by another viscous fluid. The problem is considered in the limit of large Ekman number and small Reynolds number. The analysis is carried out by combining asymptotic analysis and full numerical simulation by means of the boundary element method. We pay special attention to the stability/instability of equilibrium shapes and the possible formation of singularities representing a change in the topology of the fluid domain. When the evolution is at constant $\Omega$, depending on its value, drops can take the form of a flat film whose thickness goes to zero in finite time or an elongated filament that extends indefinitely. When evolution takes place at constant $L$ and axial symmetry is imposed, thin films surrounded by a toroidal rim can develop, but the film thickness does not vanish in finite time. When axial symmetry is not imposed and $L$ is sufficiently large, drops break axial symmetry and, depending on the value of $L$, reach an equilibrium configuration with a 2-fold symmetry or break up into several drops with a 2- or 3-fold symmetry. The mechanism of breakup is also described.
\end{abstract}

Key words. rotating drops at low Reynolds number, boundary element method, drop breakup, self-similarity and singularities

AMS subject classifications. 76D07, 65Z05

DOI. $10.1137 / 100817668$

1. Introduction. In many processes one has to deal with rotating masses of fluid. This is the case for industrial applications such as polymer manufacturing [20] and spinning drop tensiometry techniques used to measure surface and interfacial tension [24]. At the atomic level, a model for nuclear fission as the breakup of a charged rotating liquid drop, where nuclear forces play the role of surface tension, was proposed by Bohr and Wheeler in 1939 [4]. On astronomical scales, the shapes and stability of self-gravitating masses rotating freely in space were studied in detail by Chandrasekhar [7].

Studies concerning the evolution of rotating drops date back to the original experiments of Plateau [21]. In those experiments, an oil drop was immersed in a tank containing a mixture of water and alcohol with almost the same density as oil. By inserting a shaft through the drop and turning it, rotation on the drop was achieved. Plateau then observed how the drop evolved through a sequence of different shapes as the angular speed was increased. In recent years, experiments aimed in the same direction as the ones performed by Plateau in 1843 have been conducted under zero gravity conditions during the flight of Spacelab 3 and at JPL (the Jet Propulsion Laboratory). For a fully detailed description of these experiments, see, for instance, [28] and [29].

Computation of equilibrium shapes for this problem was initiated by Poincaré in 1885 [22]. He showed that two families of solutions, one of which consists of axisymmetric shapes and the other of asymmetric (2-fold shapes), could be obtained.

*Received by the editors December 9, 2010; accepted for publication (in revised form) July 25, 2011; published electronically November 22, 2011. This research was supported by the project MTM2008-03255 from the Ministerio de Ciencia e Innovación of Spain.

http://www.siam.org/journals/siap/71-6/81766.html

†Instituto de Ciencias Matemáticas (CSIC - UAM - UC3M - UCM), C/Nicolás Cabrera, 13-15, Campus de Cantoblanco, 28049 Madrid, Spain (marco.fontelos@uam.es, victor.garcia@icmat.es).

‡Departamento de Matemática Aplicada y Met. Inf., Universidad Politécnica de Madrid, Alenza 4, 28003 Madrid, Spain (ultano.kindelan@upm.es).

1941 
After Poincaré, the determination of new equilibrium solutions has been the subject of many studies. Brown and Scriven [6] determined branches of solutions where the axial symmetry of the drop is broken and drops present a 2 -fold, 3 -fold, or in general $n$-fold symmetry. The drops with 2 -fold symmetry can be observed, for instance, when a drop that is rolling over an inclined plane leaves the plane, undergoes free fall [1], and then presents a so-called peanut shape. Remarkably, the branches of solutions with $n$-fold symmetry also contain solutions such that each lobe consists of a chain of small droplets connected by thin filaments, as demonstrated by Heine [14]. In addition to equilibrium shapes with the topology of a sphere, there are also equilibrium shapes with the form of a torus [13], [26].

Although there is a huge wealth of equilibrium shapes, one cannot expect that all of them will be stable. Once a rotating drop in equilibrium destabilizes, there are essentially two possibilities: the drop may undergo a transition towards another equilibrium shape, or it may evolve in such a way that its surface becomes nonsmooth at some time and a singularity, whether representing a surface cusp or a topological change in the drop (via breakup into smaller drops, for instance), develops. The fact that singularities may take place in free-surface hydrodynamic flows is well known and has been the subject of intensive research in various contexts such as capillary drop breakup or air entrainment. (See [11] for an updated general review on free-surface flows, and [10] for a review on singularities in general.)

In order to compute the evolution of a viscous drop under rotation, one must solve the Navier-Stokes equations both inside the drop and in the fluid surrounding it, subject to suitable boundary conditions. If the Reynolds number is small, then inertial terms can be neglected in comparison with both viscous forces and centrifugal forces, and one arrives then at a Stokes system subject to centrifugal forces. This is the approach adopted by Howell, Scheid, and Stone [15] for studying the evolution and possible singularity formation of an axisymmetric drop rotating with constant angular velocity. Under a thin film approximation, those authors show that, for sufficiently large angular velocities, drops may become very flat and thin at the center with a torus-like boundary (a pizza shape, in the notation of [15]). Moreover, they find that the thickness of the center can become zero in finite time so that a hole develops.

When dealing with the evolution of a fluid at small Reynolds number the approximation by a Stokes system is justified. In this case, the fluid dynamics equations become linear, and one can write the problem in the form of the so-called boundary integral formulation (cf. [23]). This is very suitable for numerical simulation since it allows one to compute the velocity field at the boundary of the drop, and hence the evolution of the drop's shape, by evaluating integrals restricted to the boundary itself (see [23] or [5], for instance). The boundary integral formulation is particularly interesting when dealing with the possible formation of singularities, like those appearing in the evolution of charged droplets [3], [12].

In this article we implement a boundary element method to compute the evolution of drops rotating around a fixed axis, determine the stability/instability of equilibrium shapes, and study the formation of singularities that give rise to topological changes in the drop. In section 2 we introduce the system of equations that we are going to solve. In section 3, the details of the numerical method used are explained. Since we aim to analyze singularities that develop at the drop's surface, special attention must be paid to local refinement and regularization of the mesh. In section 4 we study the evolution of axisymmetric drops. Section 5 will be devoted to the study of general three-dimensional drops at both constant angular velocity and constant angular momentum. Finally, in section 6 we summarize the conclusions of our work. 
2. Equations. Consider two viscous incompressible fluids: one for the drop and another for its surrounding media. The fluid drop has viscosity $\mu_{1}$ and density $\rho_{1}$, while the surrounding fluid has viscosity $\mu_{2}$ and density $\rho_{2}$. We denote the velocity and pressure of the fluid inside the drop by $\mathbf{u}^{(1)}$ and $p^{(1)}$, respectively, and the velocity and pressure of the outer fluid by $\mathbf{u}^{(2)}$ and $p^{(2)}$, respectively. Both fluids are rotating around a common axis with angular velocity $\boldsymbol{\omega}$. In this situation, one can take a system of reference that is rotating with the fluids (noninertial frame) and therefore introduce inertial forces into the Navier-Stokes equations so that

(1)

$\rho_{i}\left(\mathbf{u}_{t}^{(i)}+\mathbf{u}^{(i)} \cdot \nabla \mathbf{u}^{(i)}\right)=-\nabla p^{(i)}+\mu_{i} \Delta \mathbf{u}^{(i)}-2 \rho_{i} \boldsymbol{\omega} \times \mathbf{u}^{(i)}-\rho_{i} \boldsymbol{\omega} \times(\boldsymbol{\omega} \times \mathbf{r})$ in $\mathcal{D}_{i}(t)$,

$$
\nabla \cdot \mathbf{u}^{(i)}=0 \text { in } \mathcal{D}_{i}(t)
$$

where $\mathcal{D}_{1}(t)$ is the domain enclosed by the fluid drop and $\mathcal{D}_{2}(t)$ is the domain of the surrounding fluid. The third and fourth terms on the right-hand side of (1) are called the Coriolis and centrifugal forces, respectively. As boundary conditions, we impose balance of stresses across the interface of both fluids:

$$
\left(T^{(2)}-T^{(1)}\right) \mathbf{n}=2 \gamma \kappa \mathbf{n} \quad \text { on } \partial \mathcal{D}(t),
$$

where $\kappa$ is the mean curvature of the interface, i.e., the average of principal curvatures; $\gamma$ is the surface tension; and $T^{(k)}$ is the stress tensor inside $(k=1)$ and outside $(k=2)$ the drop, given by

$$
T_{i j}^{(k)}=-p^{(k)} \delta_{i j}+\mu_{k}\left(\frac{\partial u_{i}^{(k)}}{\partial x_{j}}+\frac{\partial u_{j}^{(k)}}{\partial x_{i}}\right), \quad k=1,2 .
$$

Equation (3) expresses the balance between viscous stresses and capillary forces at the interface. The normal component of the velocity has to be continuous across the boundary

$$
\mathbf{u}^{(1)} \cdot \mathbf{n}=\mathbf{u}^{(2)} \cdot \mathbf{n} \equiv \mathbf{u} \cdot \mathbf{n},
$$

where $\mathbf{n}$ is the unit normal to $\partial \mathcal{D}(t)$ pointing outward from the fluid drop. The kinematic condition is

$$
v_{N}=\mathbf{u} \cdot \mathbf{n} \text { on } \partial \mathcal{D}(t),
$$

$v_{N}$ being the normal velocity of the free boundary $\partial \mathcal{D}(t)$.

If the axis of rotation is fixed, then

$$
\boldsymbol{\omega} \times(\boldsymbol{\omega} \times \mathbf{r})=-\omega^{2} r \mathbf{e}_{\mathbf{r}},
$$

where $\omega=|\boldsymbol{\omega}|$ is the angular speed, $r$ is the distance from the point located at $\mathbf{r}$ to the axis of rotation, and $\mathbf{e}_{\mathbf{r}}$ is the polar radial vector. Notice that the centrifugal force $-\rho_{i} \boldsymbol{\omega} \times(\boldsymbol{\omega} \times \mathbf{r})$ is then directed in the outward radial direction. It is useful to write the centrifugal force in the form

$$
\rho_{i} \omega^{2} r \mathbf{e}_{\mathbf{r}}=\nabla\left(\frac{1}{2} \rho_{i} \omega^{2} r^{2}\right),
$$

since the centrifugal force is conservative, and define

$$
\Pi^{(i)}=p^{(i)}-\frac{1}{2} \rho_{i} \omega^{2} r^{2}
$$

Copyright $@$ by SIAM. Unauthorized reproduction of this article is prohibited. 
as the reduced pressure. Then, system (1)-(2) becomes

$$
\begin{aligned}
\rho_{i}\left(\mathbf{u}_{t}^{(i)}+\mathbf{u}^{(i)} \cdot \nabla \mathbf{u}^{(i)}\right) & =-\nabla \Pi^{(i)}+\mu_{i} \Delta \mathbf{u}^{(i)}-2 \rho_{i} \boldsymbol{\omega} \times \mathbf{u}^{(i)} \text { in } \mathcal{D}_{i}(t), \\
\nabla \cdot \mathbf{u}^{(i)} & =0 \text { in } \mathcal{D}_{i}(t),
\end{aligned}
$$

and boundary condition (3) can be written as

$$
\left(T^{(2)}-T^{(1)}\right) \mathbf{n}=\left(2 \gamma \kappa+\frac{\left(\rho_{2}-\rho_{1}\right)}{2} \omega^{2} r^{2}\right) \mathbf{n} \quad \text { on } \partial \mathcal{D}(t),
$$

where we take now the following definition for $T^{(k)}$ :

$$
T_{i j}^{(k)}=-\Pi^{(k)} \delta_{i j}+\mu_{k}\left(\frac{\partial u_{i}^{(k)}}{\partial x_{j}}+\frac{\partial u_{j}^{(k)}}{\partial x_{i}}\right), \quad k=1,2,
$$

instead of (4). In order to nondimensionalize the system (6)-(7) we introduce a characteristic length $l$ (of the order of the radius of the drop), a typical velocity $U$ ( $\omega l$, for instance), and a characteristic time scale $\tau=l / U$. We will also suppose without loss of generality that the axis of rotation is the $z$ axis. Taking $\mu$ and $\rho$ to be reference values for the viscosity and density of the fluids, respectively, (we can take them as those of the drop) and defining the new variables

$$
\overline{\mathbf{u}}^{(i)}=\frac{\mathbf{u}^{(i)}}{U}, \quad \overline{\mathbf{r}}=\frac{\mathbf{r}}{l}, \quad \bar{t}=\frac{t}{\tau}, \quad \bar{\Pi}^{(i)}=\frac{l}{\mu U} \Pi^{(i)}, \quad \boldsymbol{\omega}=\omega \widehat{\mathbf{z}}, \quad \bar{\mu}_{i}=\frac{\mu_{i}}{\mu}, \quad \bar{\rho}_{i}=\frac{\rho_{i}}{\rho},
$$

we get, omitting overbars to simplify notation, the nondimensional problem

$$
\begin{aligned}
\rho_{i} R e\left(\mathbf{u}_{t}^{(i)}+\mathbf{u}^{(i)} \cdot \nabla \mathbf{u}^{(i)}\right) & =-\nabla \Pi^{(i)}+\mu_{i} \Delta \mathbf{u}^{(i)}-\frac{2}{E k} \widehat{\mathbf{z}} \times \mathbf{u}^{(i)} \text { in } \mathcal{D}_{i}(t), \\
\nabla \cdot \mathbf{u}^{(i)} & =0 \text { in } \mathcal{D}_{i}(t) .
\end{aligned}
$$

Two dimensionless parameters arise: $R e$ is the Reynolds number (measuring the relative importance between inertial and viscous forces), and $E k$ is the Ekman number (characterizing the relation between Coriolis and viscous forces). They are defined as

$$
R e=\frac{\rho U l}{\mu}, \quad E k=\frac{\mu}{\rho \omega l^{2}},
$$

To complete the nondimensionalization process, one has to scale the boundary condition. If we define

$$
\bar{\kappa}=l \kappa, \quad \bar{r}=\frac{r}{l}, \quad \bar{T}^{(k)}=\frac{l}{\mu U} T^{(k)},
$$

then (8) can be written as

$$
\left(T^{(2)}-T^{(1)}\right) \mathbf{n}=\frac{1}{C a}\left(2 \kappa-\frac{B o}{2} r^{2}\right) \mathbf{n} \quad \text { on } \partial \mathcal{D}(t),
$$

where overbars are omitted as before. Two new dimensionless parameters come into play, $C a$ and $B o$, called the capillary number and rotational Bond number, respectively:

$$
C a=\frac{\mu U}{\gamma}, \quad B o=\frac{\left(\rho_{1}-\rho_{2}\right) \omega^{2} l^{3}}{\gamma}
$$

Copyright $@$ by SIAM. Unauthorized reproduction of this article is prohibited. 
The first measures the importance of viscous relative to capillary forces, and the second the importance of centrifugal forces relative to capillary forces. We take as a characteristic length scale of the problem, for instance, $l=\sqrt[3]{V o l}$, with $V o l$ being the volume of the drop, and a dimensionless angular velocity defined as $\Omega=\sqrt{B o}$ to describe our results. Dealing with the limit in which $R e \ll 1$ and $E k \gg 1$, so that viscous forces dominate over inertial and Coriolis forces, we can approximate the Navier-Stokes equations by the Stokes system

$$
\begin{aligned}
-\nabla \Pi^{(i)}+\mu_{i} \Delta \mathbf{u}^{(i)}=0 & \text { in } \mathcal{D}_{i}(t), \\
\nabla \cdot \mathbf{u}^{(i)}=0 & \text { in } \mathcal{D}_{i}(t),
\end{aligned}
$$

which is numerically solved in the next section using the boundary element method. Since (11)-(12) together with the boundary condition (10) form a linear system, one can remove the dependence on $C a$ by simply rescaling again the unknowns $\mathbf{u}^{(i)}, \Pi^{(i)}$. Therefore, without loss of generality we will consider $C a=1$.

Concerning the stationary problem (where $\mathbf{u}^{(i)} \equiv \mathbf{0}$ ), equilibrium solutions can be calculated by solving the following differential equation:

$$
2 \kappa=-\Pi+\frac{B o}{2} r^{2} \text { on } \partial \mathcal{D},
$$

where $\Pi=\Pi_{2}-\Pi_{1}$ is the pressure difference sustained across the interface of both fluids. It is interesting to point out that this differential equation for equilibrium shapes can be obtained by invoking a variational argument (cf. [7], [6]). In this variational approach, an energy functional defined by taking into account the surface energy and the rotational kinetic energy of the drop is minimized subject to a volume preservation constraint. The energy to be minimized is defined in two different ways depending on the kind of problem considered. If we have a drop rotating with constant angular speed $\Omega$, then the energy of the system is

$$
\mathcal{E}=A-\frac{1}{2} I \Omega^{2}
$$

where $A$ is the area of the surface $\partial \mathcal{D}$ and $I$ is the moment of inertia of the drop, defined by the formula

$$
I=\int_{\mathcal{D}} r^{2} d V
$$

with $r$ being the distance from a point located at $\mathbf{r} \in \mathcal{D}$ to the axis of rotation. On the other hand, if the drop is mechanically isolated (angular momentum is constant), the energy functional is given by

$$
\mathcal{E}=A+\frac{1}{2} I \Omega^{2}=A+\frac{L^{2}}{2 I}
$$

where $L=I \Omega$ is the angular momentum of the fluid drop. Finally, we remark that our nondimensionalization is slightly different from that of [6], since we aimed to obtain the expressions (14) and (16) that we believe are physically more intuitive. There is a simple relation between our values of $(\Omega, L)$ and those of [6], denoted by $\left(\Omega_{B S}, L_{B S}\right)$ : $(\Omega, L)=\left(4 \sqrt{\frac{2 \pi}{3}} \Omega_{B S}, 8 \sqrt{2}\left(\frac{3}{4 \pi}\right)^{7 / 6} L_{B S}\right)$. 
3. Numerical method. Our interest in the evolution of the drop's surface suggests the use of the boundary element method (BEM) to calculate the velocity at the interface between the two fluids. The BEM we have implemented is based on the boundary integral formulation of the Stokes system (11) with the boundary condition (10) (see [23], [25] for a comprehensive explanation). According to this, the equation for the velocity at $\partial \mathcal{D}(t)$ can be written as

$$
\begin{aligned}
u_{j}\left(\mathbf{r}_{p}\right)= & -\frac{1}{4 \pi} \frac{1}{\mu_{1}+\mu_{2}} \int_{\partial \mathcal{D}(t)} f_{i}(\mathbf{r}) G_{i j}\left(\mathbf{r}, \mathbf{r}_{p}\right) d S(\mathbf{r}) \\
& -\frac{1}{4 \pi} \frac{\mu_{1}-\mu_{2}}{\mu_{2}+\mu_{1}} \int_{\partial \mathcal{D}(t)} u_{i}(\mathbf{r}) T_{i j k}\left(\mathbf{r}, \mathbf{r}_{p}\right) n_{k}(\mathbf{r}) d S(\mathbf{r}), \quad i, j, k=1,2,3
\end{aligned}
$$

where $\mathbf{r}_{p}$ is the position vector of a point $p$ of the surface, and

$$
\begin{aligned}
G_{i j}\left(\mathbf{r}, \mathbf{r}_{p}\right) & =\frac{\delta_{i j}}{\left|\mathbf{r}-\mathbf{r}_{p}\right|}+\frac{\left(r_{i}-r_{p, i}\right)\left(r_{j}-r_{p, j}\right)}{\left|\mathbf{r}-\mathbf{r}_{p}\right|^{3}}, \quad i, j, k=1,2,3, \\
T_{i j k}\left(\mathbf{r}, \mathbf{r}_{p}\right) & =-6 \frac{\left(r_{i}-r_{p, i}\right)\left(r_{j}-r_{p, j}\right)\left(r_{k}-r_{p, k}\right)}{\left|\mathbf{r}-\mathbf{r}_{p}\right|^{5}}, \quad i, j, k=1,2,3, \\
f_{i}(\mathbf{r}) & =\left[2 \kappa(\mathbf{r})-\frac{1}{2} \Omega^{2} r^{2}\right] n_{i}(\mathbf{r}), \quad i=1,2,3 .
\end{aligned}
$$

We remark that, by using (17), we are assuming that the velocity of the outer fluid at infinity is zero in our frame of reference that is rotating with angular velocity $\Omega$. This would correspond, for instance, to the physical situation when both fluids are inside a container that is rotating with angular velocity $\Omega$. A different situation arises if one considers that the ambient fluid is not rotating at infinity, which, in the frame of reference rotating with $\Omega$, is equivalent to saying that $\mathbf{u}^{(2)} \sim \mathbf{u}^{\infty}(\mathbf{r}) \equiv-(\boldsymbol{\Omega} \times \mathbf{r})$ as $|\mathbf{r}| \rightarrow \infty$. This would add a new term $2 \mu_{2} u_{j}^{\infty}(\mathbf{r}) /\left(\mu_{1}+\mu_{2}\right)$ on the right-hand side of (17). When $\mu_{2} / \mu_{1} \ll 1$, the outer fluid produces a negligible viscous traction on the drop's surface (this is the case of water surrounded by air or oil surrounded by water, for instance) and this new term can be safely neglected. We then return to (17), which is the equation that will be considered in this paper.

At any given time $t>0$, we approximate the free boundary $\partial \mathcal{D}(t)$ with a triangular mesh. The mesh is made up of $N$ vertices and $M$ (triangular) faces. On each node, we approximate the various physical quantities that are defined on the surface (centrifugal force, curvature, velocity) with elementwise constant functions over a "virtual" element centered in each node with an area equal to one third of the total area of the elements that share the node (see [30]). We also use the nodes of the mesh as collocation points.

The general description of how we solve the integral equations is as follows: first we calculate the curvature $\kappa$ in each node of the mesh, and second we calculate the velocity by obtaining the balance force term $\mathbf{f}$ from the centrifugal force and $\kappa$, replacing it in (17), and solving the resulting integral equation. Given the velocity $\mathbf{u}$, we move the points of the surface using the explicit Euler scheme and regularize the mesh. In the next subsections we will describe the BEM used to compute the velocity field at the surface of the drop (including the regularization and refinement of the mesh) and the two procedures we have implemented for computing the mean curvature of the drop's surface and the moment of inertia of the drop. We have used two codes based on the general principles sketched above. In the simplest one, axial 
symmetry is assumed, and explicit integration of the boundary integral in the polar coordinate $\theta$ is performed. The other code does not assume any kind of symmetry and is the one we explain in more detail in this section; the axisymmetric code is a simplified version of it, and so we briefly describe it at the end of the section.

3.1. Mean curvature. We calculate the mean curvature in each node $p$ of the mesh following a method proposed in [30]. The algorithm is based on the following idea. If the $z^{\prime}$ axis of the local Cartesian coordinates $\left(\mathbf{r}_{p}, x^{\prime}, y^{\prime}, z^{\prime}\right)$ was directed along the normal vector $\mathbf{n}_{p}$, then $z^{\prime}$ as a quadratic function of $x^{\prime}$ and $y^{\prime}$ would be a good local representation of the surface $\partial \mathcal{D}$. This quadratic function can be obtained by finding a paraboloid which passes through $p$, has its axis parallel to $z^{\prime}$, and fits best its neighbors by the least-squares method. However, $\mathbf{n}_{p}$ is not known a priori, and so the method is iterative. The formal description of this method appears in Algorithm 1.

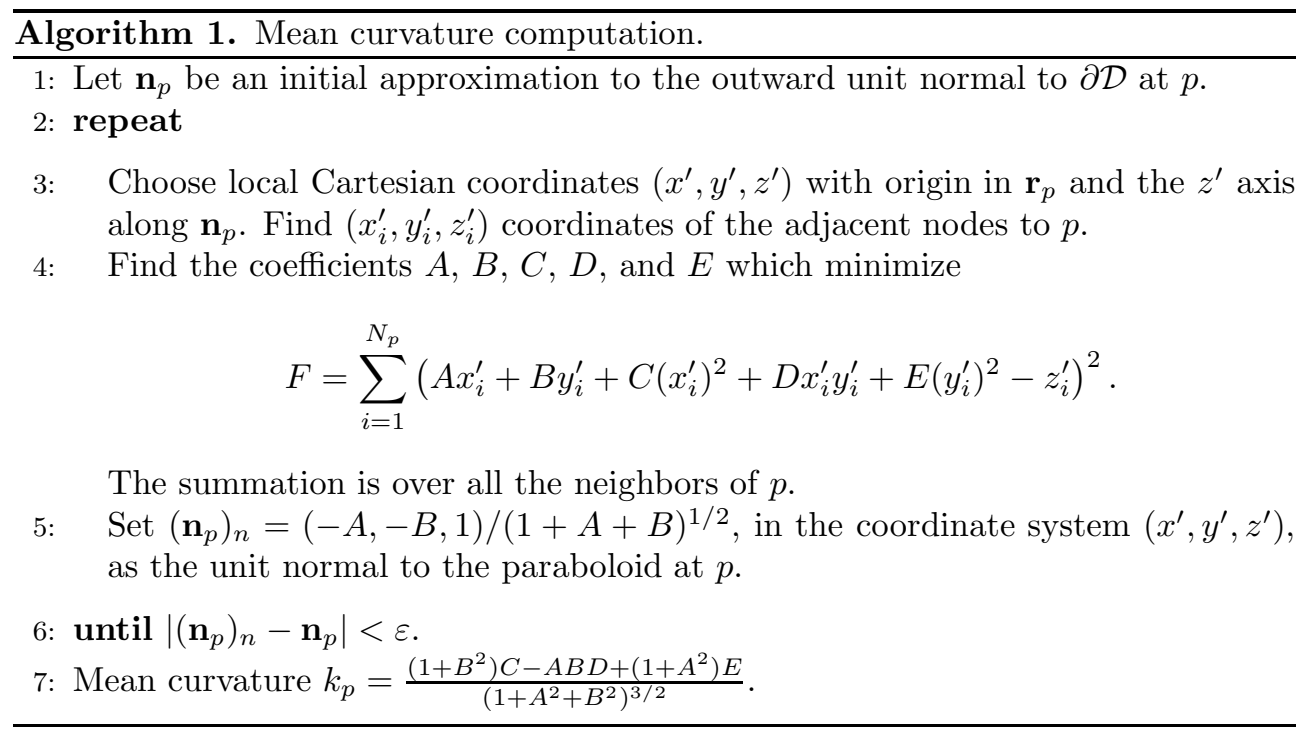

Note that, due to the use of the least-squares method, each node of the mesh must have five or more neighbors. The convergence is very fast with only a few iterations needed, even for $\varepsilon \sim 10^{-6}-10^{-8}$. We also use this algorithm to calculate the normal vector to the surface at each node of the mesh.

3.2. Velocity. Once the curvature and the centrifugal force are known in each node of the mesh we are able to evaluate the balance force term $\mathbf{f}$ and thus, replacing $\mathbf{f}$ in (17), calculate $\mathbf{u}$ in each node of the mesh.

The first integral in (17) is known as the single layer potential, whereas the second one is termed the double layer potential. Both integrals are singular in $\mathbf{r}=\mathbf{r}_{p}$. We will remove both singularities with a well-known technique proposed, for example, in [23], which is based on the fact that

$$
\int_{\partial \mathcal{D}(t)} G_{i j}\left(\mathbf{r}, \mathbf{r}_{p}\right) n_{i}(\mathbf{r}) d S(\mathbf{r})=0 \text { and } \int_{\partial \mathcal{D}(t)} T_{i j k}\left(\mathbf{r}, \mathbf{r}_{p}\right) n_{k}(\mathbf{r}) d S(\mathbf{r})=-4 \pi \delta_{i j} .
$$

Using (21), we get an equation equivalent to (17):

$$
\begin{aligned}
u_{j}\left(\mathbf{r}_{p}\right)= & -\lambda_{S} \int_{\partial \mathcal{D}(t)}\left(b(\mathbf{r})-b\left(\mathbf{r}_{p}\right)\right) n_{i}(\mathbf{r}) G_{i j}\left(\mathbf{r}, \mathbf{r}_{p}\right) d S(\mathbf{r}) \\
& -\lambda_{D} \int_{\partial \mathcal{D}(t)}\left(u_{i}(\mathbf{r})-u_{i}\left(\mathbf{r}_{p}\right)\right) T_{i j k}\left(\mathbf{r}, \mathbf{r}_{p}\right) n_{k}(\mathbf{r}) d S(\mathbf{r})+4 \lambda_{D} \pi u_{i}\left(\mathbf{r}_{p}\right) \delta_{i j}
\end{aligned}
$$


where $\lambda_{S}=\frac{1}{4 \pi} \frac{1}{\mu_{1}+\mu_{2}}, \lambda_{D}=\frac{1}{4 \pi} \frac{\mu_{1}-\mu_{2}}{\mu_{2}+\mu_{1}}$, and $\mathbf{f}(\mathbf{r})=b(\mathbf{r}) \mathbf{n}(\mathbf{r})$. The right-hand-side integrals of (22) are approximated by a trapezoidal rule that requires the integrands only at nodes of the mesh: $\int_{\partial \mathcal{D}} \phi(\mathbf{x}) d s(\mathbf{x})=\sum_{i=1}^{N} \phi\left(\mathbf{x}_{i}\right) S_{i}$, where $S_{i}$ is the area of the virtual element associated with node $i$. Applying this, we get the discretization of (22):

$$
\begin{aligned}
& \left(1-4 \pi \lambda_{D}\right) u_{j}\left(\mathbf{r}_{p}\right)+\lambda_{D} \sum_{\substack{l=1 \\
l \neq p}}^{N}\left(u_{i}\left(\mathbf{r}_{l}\right)-u_{i}\left(\mathbf{r}_{p}\right)\right) T_{i j k}\left(\mathbf{r}_{l}, \mathbf{r}_{p}\right) n_{k}\left(\mathbf{r}_{l}\right) S_{l} \\
& =-\lambda_{S} \sum_{\substack{l=1 \\
l \neq p}}^{N}\left(b\left(\mathbf{r}_{l}\right)-b\left(\mathbf{r}_{p}\right)\right) n_{i}\left(\mathbf{r}_{l}\right) G_{i j}\left(\mathbf{r}_{l}, \mathbf{r}_{p}\right) S_{l} .
\end{aligned}
$$

Equation (23) for all values of $p \in\{1, \ldots, N\}$ and $j \in\{1,2,3\}$ forms a linear system of $3 N$ equations in the $3 N$ unknown velocity components $u_{j}\left(\mathbf{r}_{p}\right)$ at the nodes. After solving the system, we move the nodes of the surface with an Euler explicit scheme:

$$
\mathbf{r}_{i}\left(t_{n+1}\right)=\mathbf{r}_{i}\left(t_{n}\right)+\mathbf{u}_{i}\left(t_{n}\right) \triangle t, \quad i=1, \ldots, p .
$$

This process is repeated until we reach a desired time $t_{\max }$ or some other condition is met. In practice we have found that the time step $\Delta t$ cannot be arbitrarily large [27]. Instead, $\Delta t$ should be such that the displacement of any node during a single time step is smaller than the length of any edge of the mesh that includes the node. In addition, the time step should be of the same order with the characteristic time $\tau=\frac{R \mu}{\gamma}$, where $R$ is the maximum (linear) dimension of the droplet, $\mu$ is the minimum of the two viscosities $\mu_{1,2}$, and $\gamma$ is the capillarity constant.

3.3. Moment of inertia. When dealing with the evolution of droplets at constant angular momentum, one needs to compute the moment of inertia of a solid whose boundary is a triangle mesh. A simple and efficient numerical algorithm, which is described in detail in [16], is briefly introduced here for this purpose. The moment of inertia about the $z$ axis of a fluid drop with constant density $\rho_{1}$ is given by the formula

$$
I_{z z}=\rho_{1} \int_{\mathcal{D}_{1}(t)}\left(x^{2}+y^{2}\right) d V
$$

where $\mathcal{D}_{1}(t)$ is the region occupied by the drop. To calculate this integral consider $\mathcal{P}(t)$ as the polyhedral solid approximation of $\mathcal{D}_{1}(t)$ whose facets are triangles. Thus, $\mathcal{P}(t)$ can be conceived as a signed sum of tetrahedra, $\mathcal{C}_{\mathcal{P}}(t)$, where its elements are constructed from the origin to the vertices of each of the triangle facets. The sign of the contribution for each tetrahedron $\mathcal{T}, \epsilon(\mathcal{T})$, is determined by the sign of the dot product between the barycenter of the facet and the outward unit normal vector to $\partial \mathcal{P}(t)$ at that facet. This yields the following approximation:

$$
I_{z z}=\rho_{1} \int_{\mathcal{D}_{1}(t)}\left(x^{2}+y^{2}\right) d V \approx \rho_{1} \sum_{\mathcal{T} \in \mathcal{C}_{\mathcal{P}}(t)} \int_{\mathcal{T}}\left(x^{2}+y^{2}\right) d V
$$

which can be precisely computed, as the integral of a homogeneous quadratic polynomial $f(x, y, z)$ (in our case $\left.f(x, y, z)=x^{2}+y^{2}\right)$ over a tetrahedron $\mathcal{T} \in \mathcal{C}_{\mathcal{P}}(t)$ with vertices $\left(V_{1}^{\mathcal{T}}, V_{2}^{\mathcal{T}}, V_{3}^{\mathcal{T}}\right)$ verifies:

$$
\int_{\mathcal{T}} f(x, y, z) d V=\frac{\nu(\mathcal{T})}{20}\left(f\left(V_{1}^{\mathcal{T}}\right)+f\left(V_{2}^{\mathcal{T}}\right)+f\left(V_{3}^{\mathcal{T}}\right)+f\left(V_{1}^{\mathcal{T}}+V_{2}^{\mathcal{T}}+V_{3}^{\mathcal{T}}\right)\right)
$$


where $\nu(\mathcal{T})$ is the signed volume of the tetrahedron $\mathcal{T}$ :

$$
\nu(\mathcal{T})=\epsilon(\mathcal{T}) \frac{\left|\operatorname{det}\left(V_{1}^{\mathcal{T}}, V_{2}^{\mathcal{T}}, V_{3}^{\mathcal{T}}\right)\right|}{6} .
$$

Note that with this method, one can also easily calculate the volume of solids by applying

$$
\operatorname{Vol}\left(\mathcal{D}_{1}(t)\right)=\rho_{1} \int_{\mathcal{D}_{1}(t)} d V \approx \rho_{1} \sum_{\mathcal{T} \in \mathcal{C}_{\mathcal{P}}(t)} \nu(\mathcal{T}) .
$$

3.4. Regularization of the mesh. If one evolves the free boundary by applying the procedure described in the previous sections, one observes that the capability of the mesh to approximate the free boundary might deteriorate rapidly in time, especially in the cases where the geometry of the interface changes radically, for instance in the formation of cones, jets, or necks. For this reason, in our code we apply a number of mesh regularization techniques (based on previous works by Cristini, Blawzdziewicz, and Loewenberg [9] and Vantzos [27]) that allow the mesh to adapt to the evolving geometry of the interface. In more quantitative terms, we have used the fact that a suitable measure of the "quality" of a mesh is the comparison between the length of any edge with the local (minimal) radius of curvature [9].

The first regularization technique, called Delaunay remeshing, is based on the fact that, given a set of nodes, which are assumed to lie on the free boundary, there are many ways to connect them in order to form an approximating mesh. The objective is to find the mesh that has a minimal number of "thin triangles," e.g., triangles with very small angles. This can be achieved by beginning with an initial mesh and then performing a sequence of "edge flips" (see Figure 1).

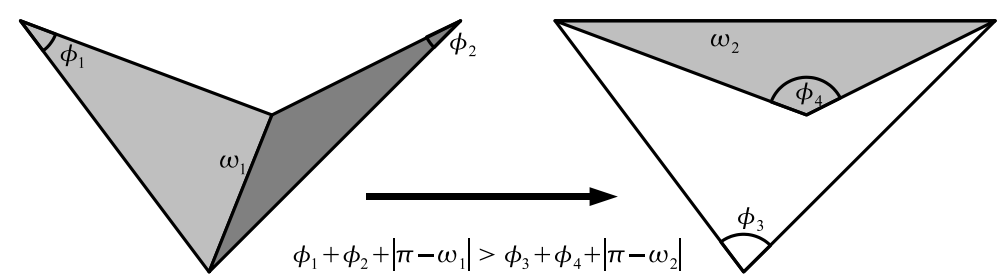

FIG. 1. Mesh restructuring is performed via a sequence of edge-flips such as the one pictured here. We exchange the diagonals of the (nonplanar) quadrilateral formed by two adjacent triangles if a certain inequality holds. Note that $\omega_{1}$ and $\omega_{2}$ are the dihedral angles of the two configurations.

The second regularization technique addresses the phenomenon that nodes need to be constantly reallocated on the surface in order to maintain a (locally) uniform distribution. This can be achieved by treating the mesh as a system of masses (the nodes), whose position is restricted on the surface and connected by springs (the edges) with an extremely high damping constant [9]. Letting the nodes move under the influence of these spring forces for a certain time interval is called "relaxing the mesh." Note that in order to have a measurable improvement of the quality of the mesh, we need to set the natural (relaxed) length of every spring equal to the local (minimal) radius of curvature.

Although relaxation does improve the distribution of the nodes, it does not address the fact that when the local curvature increases at a region of the free boundary, 
the density of the nodes must increase accordingly for the mesh to resolve the developing geometrical feature satisfyingly. This can be achieved by refining the mesh, i.e., subdividing the faces of the mesh that are "too large." A face is considered too large, and thus a candidate for subdivision, if its area is larger than a certain multiple of the area of an equilateral triangle with side equal to the local radius of curvature. The subdivision scheme that we use divides the triangular face into three new triangles by adding a new node close to the center of mass of the triangle and connecting it to the three existing vertices [27]. The new position of the new node is calculated using the osculating paraboloids from section 3.1 as a local representation of the surface. The refinement is always followed by remeshing and relaxation (see Figure 2).

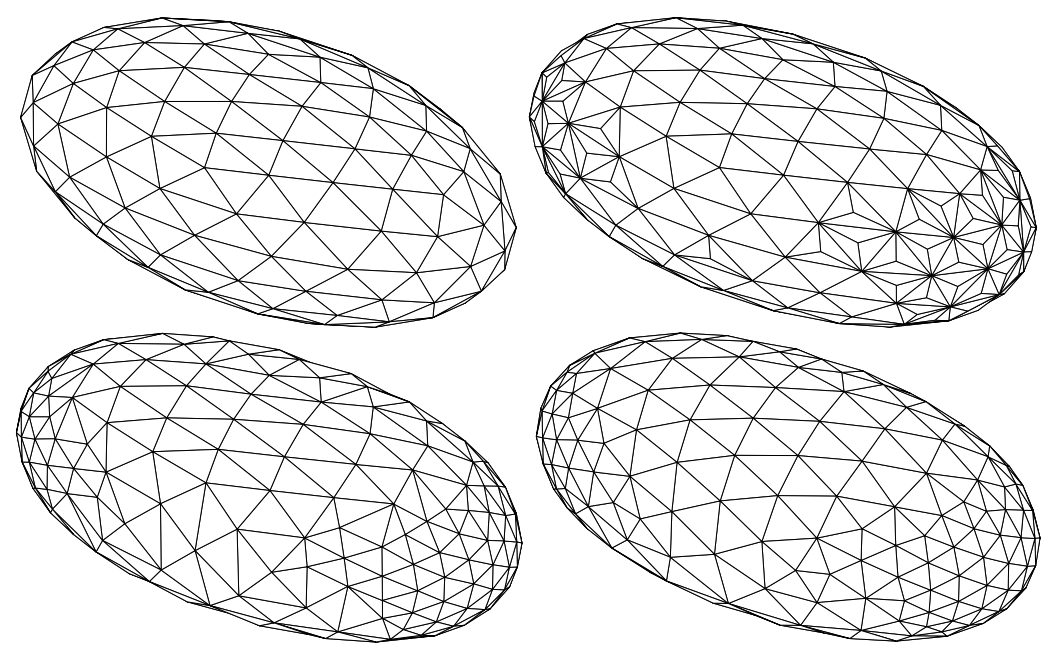

FIG. 2. Example of mesh regularization. Starting with a given mesh (top left), we refine the areas of high curvature (top right). After refining, we apply remeshing (bottom left) and relaxation (bottom right).

3.5. The code for axially symmetric configurations. The implementation of BEMs simplifies notably when one considers an axially symmetric configuration. First, the computation of the mean curvature, when the interface is written in the form $z=h(r, t)$, has an explicit formula in terms of $h(r, t)$ and its derivatives up to second order that can be computed using simple finite differences. Second, the integrals in the azimuthal angle $\theta$ that appear in (22) can be explicitly calculated as a previous step to discretizing the profile. These integrals may involve more or less complicated functions, such as elliptic integrals (cf. [23]), but are explicit in terms of them. The profile $(r, h(r, t))$ is then discretized in the form $\left\{\left(r_{i}, h\left(r_{i}, t\right)\right), i=1, \ldots, N\right\}$, and integrals are approximated in the same way as in the three-dimensional (3D) code, also using a singularity removal procedure. Nodes are moved influenced by artificial spring forces, as in the relaxation method explained above, so that they concentrate in regions of high curvature. The reduction in the dimension of the problem allows for a large number of mesh points and precise description of the "high curvature" regions up to several (typically 3 or 4 ) orders of magnitude of the curvature.

4. Evolution of axisymmetric drops. In this section we study the evolution of axisymmetric drops, both at constant angular speed and at constant angular momentum. First, we will present equilibrium solutions, already reported in the lit- 
erature [1], [2], [6], [13], [14], [26], and compare them with the equilibrium shapes obtained for long time behavior of evolving drops computed with the axisymmetric version of the boundary element algorithm. Later, we also present a stability analysis of equilibrium shapes and the possible formation of holes for drops evolving at constant $\Omega$ or constant $L$.

4.1. Equilibrium shapes. The axisymmetric equilibrium shapes can be calculated in a closed form by integrating the differential equation

$$
2 \kappa=-\Pi+\frac{B o}{2} r^{2}
$$

where $\Pi=\Pi^{(2)}-\Pi^{(1)}$ is a constant. We describe the geometry of the drop by the function $h(r)$, which gives the height of each point of the interface as a function of the distance $r$ to the axis of rotation. Under the assumption of axial symmetry,

$$
2 \kappa=-\frac{1}{r} \frac{d}{d r}\left(\frac{r h_{r}}{\sqrt{1+h_{r}^{2}}}\right),
$$

and therefore we need to solve the equation

$$
\frac{1}{r} \frac{d}{d r}\left(\frac{r h_{r}}{\sqrt{1+h_{r}^{2}}}\right)=-\frac{\Omega^{2}}{2} r^{2}+\Pi,
$$

subject to appropriate boundary conditions. If we assume a domain without holes and with a regular surface, then it is natural to impose $h_{r}(0)=0$, and (24) can be integrated once to yield

$$
\frac{h_{r}}{\sqrt{1+h_{r}^{2}}}=-\frac{\Omega^{2}}{8} r^{3}+\frac{\Pi}{2} r
$$

which can be explicitly solved in terms of elliptic functions (see [19]). If the domain presents a hole of radius $a$ near the axis, then integration of (24) leads to

$$
\frac{r h_{r}}{\sqrt{1+h_{r}^{2}}}-\frac{a h_{r}(a)}{\sqrt{1+\left(h_{r}(a)\right)^{2}}}=-\frac{\Omega^{2}}{8} r^{4}+\frac{\Pi}{2} r^{2}+\frac{\Omega^{2}}{8} a^{4}-\frac{\Pi}{2} a^{2},
$$

so that, by imposing $h_{r}(a)=+\infty$, one gets smooth toroidal solutions (that is, such that $h(r) \sim C(r-a)^{\frac{1}{2}}$ as $\left.r \rightarrow a^{+}\right)$that we will call toroidal type I solutions, satisfying

$$
\frac{h_{r}}{\sqrt{1+h_{r}^{2}}}=\frac{\left(a+\frac{\Omega^{2}}{8} a^{4}-\frac{\Pi}{2} a^{2}\right)}{r}-\frac{\Omega^{2}}{8} r^{3}+\frac{\Pi}{2} r .
$$

There is also the possibility that $h(r)=0$ for $0 \leq r<a$ and $h(a)=0$ with $h_{r}(a)=0$, so that the surface is still smooth if one considers that a zero thickness film lies in the region $0 \leq r<a$. These solutions have been thoroughly studied in [1]. We will call these solutions toroidal type II, and they satisfy

$$
\frac{h_{r}}{\sqrt{1+h_{r}^{2}}}=\frac{\left(\frac{\Omega^{2}}{8} a^{4}-\frac{\Pi}{2} a^{2}\right)}{r}-\frac{\Omega^{2}}{8} r^{3}+\frac{\Pi}{2} r .
$$




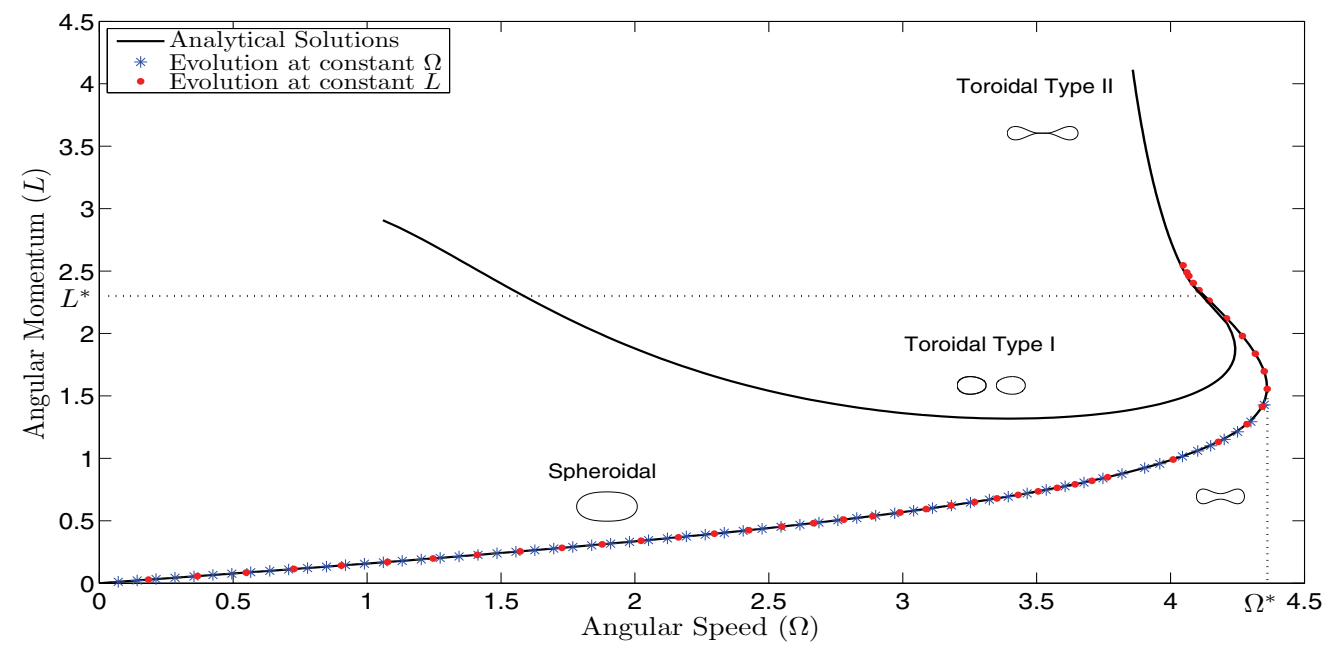

FIG. 3. Bifurcation diagram of axisymmetric equilibrium shapes. Theoretical $L-\Omega$ curves (continuous line) together with asymptotic values, for $t \rightarrow \infty$, of an initially spherical drop evolving at constant $\Omega$ (asterisks) or at constant $L$ (dots).

In Figure 3 we represent in the $L-\Omega$ plane the values of the angular momentum $L$ versus the angular velocity $\Omega$ for each of these solutions together with the asymptotic values that result when the evolution of an initially spherical drop, which we compute using the boundary element method described above, converges to one of these equilibria. Notice that equilibrium toroidal type II shapes can occur only when the evolution takes place at constant $L$, while the evolution at constant $\Omega$ can converge to spheroidal type equilibrium shapes only if $\Omega$ is below some critical value $\Omega^{*}$. We will discuss below the evolution at constant $\Omega>\Omega^{*}$, and the mechanism of convergence to toroidal shapes of type II at constant $L$ (for $L$ larger than some $L^{*}$ ) will be described at the end of this section.

4.2. Evolution at constant $\Omega$. When $\Omega$ is sufficiently small, starting from a spherical drop, the evolution leads to an equilibrium shape in the family described in Figure 3 (some of those shapes are represented in Figure 4(left)). The condition that the drop's volume is one leads, from (24), to the implicit equation

$$
\begin{aligned}
1=V o l & =4 \pi \int_{0}^{r_{\max }} h(r) r d r=-2 \pi \int_{0}^{r_{\max }} h_{r}(r) r^{2} d r \\
& =\frac{\pi}{4} \int_{0}^{r_{\max }} \frac{\Omega^{2} r^{5}-4 \Pi r^{3}}{\sqrt{1-\left(\frac{\Omega^{2}}{8} r^{3}-\frac{\Pi}{2} r\right)^{2}}} d r \equiv f(\Omega, \Pi),
\end{aligned}
$$

where $r_{\max }$ is such that $\frac{\Omega^{2}}{8} r_{\max }^{3}-\frac{\Pi}{2} r_{\max }=1$. The equation $f(\Omega, \Pi)=1$ defines $\Omega$ as a function of $\Pi$. The angular momentum can then be computed as

$$
\begin{aligned}
L=\Omega I & =4 \pi \Omega \int_{0}^{r_{\max }} h(r) r^{3} d r=-\pi \Omega \int_{0}^{r_{\max }} h_{r}(r) r^{4} d r \\
& =\frac{\pi \Omega}{8} \int_{0}^{r_{\max }} \frac{\Omega^{2} r^{7}-4 \Pi r^{5}}{\sqrt{1-\left(\frac{\Omega^{2}}{8} r^{3}-\frac{\Pi}{2} r\right)^{2}}} d r,
\end{aligned}
$$

Copyright $\odot$ by SIAM. Unauthorized reproduction of this article is prohibited. 

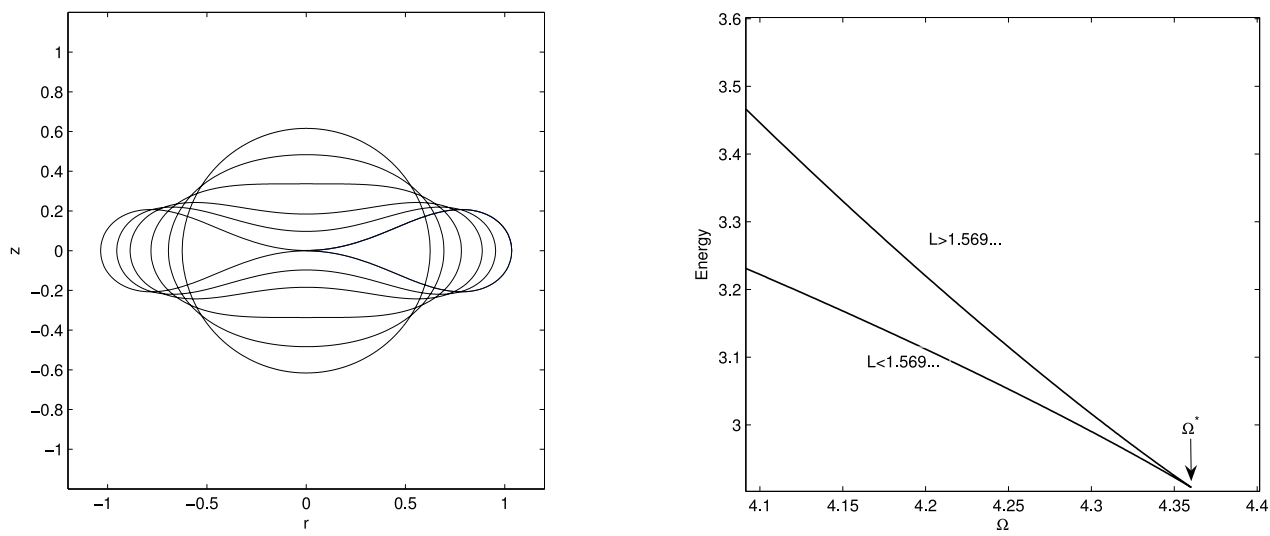

FIG. 4. Left: Equilibrium shapes for $(\Omega, L)=(0,0),(3.14,0,61),(4.09,1.05),(4.36,1.57)$, $(4.29,1.91),(4.09,2.38)$ (in order of higher eccentricity). Notice the existence of two different profiles for $\Omega=4.09$. Right: Total energy as a function of $\Omega$.

leading also to $L$ as a function of $\Pi$. With $(\Omega(\Pi), L(\Pi))$ one can represent the branch of spheroids in Figure 3. There exists a value of $\Omega$, denoted by $\Omega^{*}=4.3648 \ldots$, such that axisymmetric equilibrium shapes do not exist for $\Omega>\Omega^{*}$. (This value has been computed numerically.) Notice that for $\Omega<\Omega^{*}$ there is the possibility of two equilibrium solutions with the same $\Omega$ but different $L$. We can easily compute the energy

$$
\mathcal{E}=A-\frac{1}{2} I \Omega^{2}
$$

with

$$
A=4 \pi \int_{0}^{r_{\max }} r \sqrt{1+h_{r}^{2}(r)} d r=4 \pi \int_{0}^{r_{\max }} \frac{r}{\sqrt{1-\left(\frac{\Omega^{2}}{8} r^{3}-\frac{\Pi}{2} r\right)^{2}}} d r,
$$

and deduce (see Figure 4(right)) that those shapes with smaller values of $L$ possess less energy and hence should be more stable. In fact, our numerical simulations verify this. When $\Omega>\Omega^{*}$, the drop's surface becomes concave during the evolution. From this moment, mass is continuously evacuated from the neighborhood of the axis until a hole develops.

Once an equilibrium shape becomes unstable it undergoes evolution towards the formation of a singularity, as discussed in [15]. The singularity is such that the drop evolves into a toroidal rim of fluid with a thin film inside (see Figure 5). According to [15], the radius of the toroidal rim grows as $r_{\max }=O\left(\left(t_{0}-t\right)^{-\frac{1}{2}}\right)$, i.e., blows up in finite time. Also the minimum thickness $h_{\min }(t)$ of the inner film vanishes at $t_{0}$, and the behavior of $h_{\min }(t)$ is characterized by a second kind self-similar solution $h_{\min }(t) \sim O\left(\left(t_{0}-t\right)^{p(\Omega)}\right)$ with $p=4.1236$ in the limit $\Omega \rightarrow \infty$ (or equivalently when surface tension is neglected and Bo $\rightarrow \infty$ ). In fact, the fact that $r_{\max }$ diverges follows from the following argument in [15]. Assume that the shape consists of a disc of radius $\beta(t)$ and $r$-dependent thickness $h(r, t)$, surrounded by a toroidal rim of radius of the tube $R(t)$; then, the evolution can be described by the following thin film equations (see [15]): 


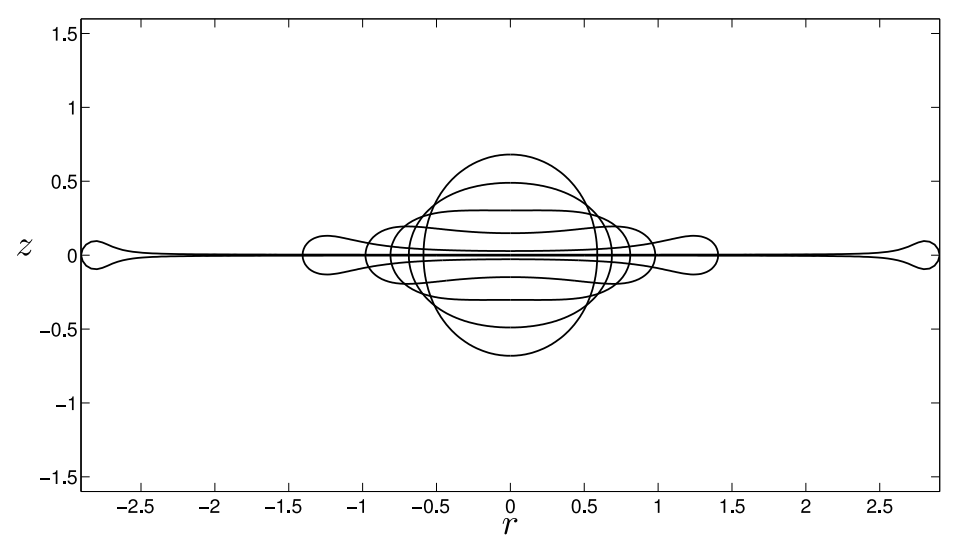

FIG. 5. Evolution of a drop at constant $\Omega=10$ for times $t=0,0.1,0.2,0.3,0.4,0.5$ and $\mu_{1}=1$, $\mu_{2}=0.1$.

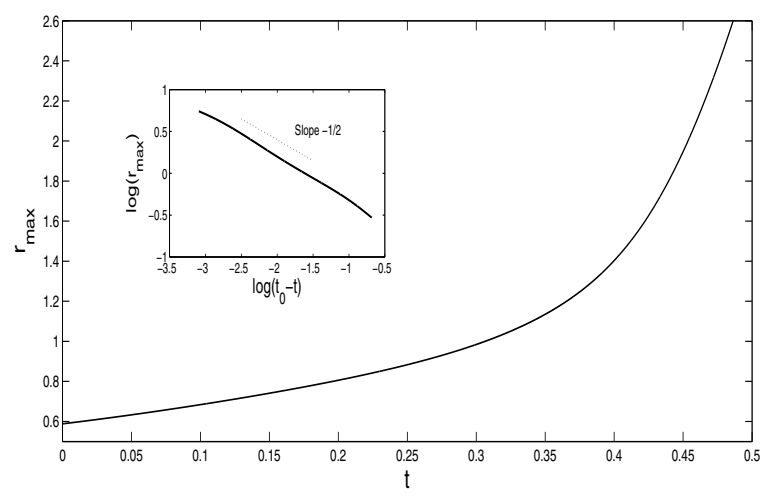

FIG. 6. Evolution of the equatorial radius of the drop at constant $\Omega=10$. Inset: $\log \left(r_{\max }\right)$ vs. $\log \left(t_{0}-t\right)$ with $t_{0} \simeq 0.51$.

$$
\begin{aligned}
\frac{\partial h}{\partial t}+\frac{1}{r} \frac{\partial}{\partial r}(r h v) & =0 \\
\frac{4}{h} \frac{\partial}{\partial r}\left(\frac{h}{r} \frac{\partial}{\partial r}(r v)\right)-2 \frac{v}{h r} \frac{\partial h}{\partial r} & =-\Omega^{2} r+\frac{2}{R(t)} \delta(r-\beta(t)),
\end{aligned}
$$

where $v(r, t)$ is the radial velocity and $\delta$ is the Dirac delta function. The last term on the right-hand side of (27) models the force that the toroidal rim exerts on the film. If we search for self-similar solutions to (26), (27), from dimensional arguments one finds that they must have the form

$$
h(r, t)=\left(t_{0}-t\right)^{p} f\left(r\left(t_{0}-t\right)^{\frac{1}{2}}\right), \quad v(r, t)=\left(t_{0}-t\right)^{-\frac{3}{2}} g\left(r\left(t_{0}-t\right)^{\frac{1}{2}}\right),
$$

where $p$ is a free parameter (depending on $\Omega$ ). The solution (28) implies that the radius of the disc $\beta(t)$ blows up at a rate $\left(t_{0}-t\right)^{-\frac{1}{2}}$. Our numerical results for the growth of the drop's size $r_{\max }$, blowing up at the same rate as $\beta(t)$, support the result of [15] for the lubrication system (26), (27) (see Figure 6) up to the maximum drop extension reachable with our method.

Copyright $@$ by SIAM. Unauthorized reproduction of this article is prohibited. 


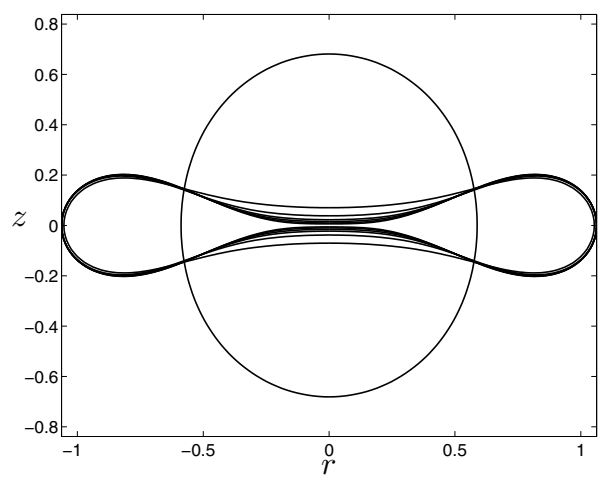

FIG. 7. Evolution of a drop at constant $L=2.54558$ for times $t=0,1, \ldots, 6$ and $\mu_{1}=1, \mu_{2}=0.1$.

It is worth noting that these solutions representing a torus escaping to infinity in finite time cannot be a valid approximation to the real situation at all times, because the velocity blows up at finite time and therefore inertial terms neglected under Stokes approximation will necessarily become dominant at some time. Then, the fluid particles will at most move with a centrifugal acceleration, so that their distance $r(t)$ to the axis will verify

$$
\frac{d^{2} r(t)}{d t^{2}} \simeq \Omega^{2} r(t)
$$

so that $r(t) \leq C e^{\Omega t}$. Once a hole appears in the inner film, it will quickly retract, and the resulting torus is subject to Plateau-Rayleigh instabilities, leading to breakup into smaller droplets. The breakup of the rotating torus will be discussed later.

4.3. Evolution at constant $\boldsymbol{L}$. When the evolution of the drop takes place at constant angular momentum, as is the case when the drop is mechanically isolated, and axial symmetry is imposed, we always converge to an equilibrium shape. Hence, all equilibrium solutions appear to be stable. There exists a subfamily of equilibrium solutions that we denote as toroidal type II (see Figure 3) consisting of a zero thickness film connecting a toroidal rim. These solutions appear for $L>L^{*}$ with $L^{*}=2.3755 \ldots$ (see Figure 7). This value for the angular momentum was determined numerically, taking into account that its angular velocity pairing in the $L-\Omega$ bifurcation diagram is analytically known [26]. A natural question is then whether the zero thickness film (that is, the formation of a hole) develops in finite or infinite time for $L>L^{*}$ and, if the hole develops in finite time, how the transition to the toroidal type I equilibrium solution with the same angular momentum takes place. Our numerical evidence is that convergence to the solutions with zero thickness film occurs at infinite time. This indicates that transition to toroidal type I solutions cannot take place. It is interesting to note that the thickness of the film follows an asymptotic law,

$$
h_{\min }(t)=h(0, t) \sim \frac{C}{\sqrt{t}},
$$


and the profiles of the interface near the point where the film and toroidal rim meet present a very clear similarity law of the form

$$
\frac{h(r, t)}{h_{\min }(t)}=f\left(\frac{r-r_{0}(t)}{\sqrt{h_{\min }(t)}}\right),
$$

where $f(\xi)$ is a similarity profile such that $f(\xi) \rightarrow 1$ as $\xi \rightarrow-\infty$ and $f(\xi) \sim A \xi^{2}$ as $\xi \rightarrow+\infty$. The radius $r_{0}(t)$ is such that $r_{0}(t) \rightarrow a$ as $t \rightarrow \infty$, where $a$ is the radius of the film.

The evolution of the inner film can be easily described by means of an explicit solution of the Stokes system that we compute next. For the sake of simplicity we restrict ourselves to the situation where no outer fluid is present. If one seeks solutions to the Stokes system in polar coordinates (cf. [17]) of the form

$$
\begin{aligned}
& u_{z}=\alpha z r^{2}+\beta z^{3}, \\
& u_{r}=\gamma r z^{2}+\delta r^{3},
\end{aligned}
$$

then it is simple to compute, from the condition $\nabla \cdot \mathbf{u}=0$, the relations

$$
\begin{array}{r}
2 \gamma+3 \beta=0, \\
4 \delta+\alpha=0,
\end{array}
$$

so that

$$
\begin{aligned}
& p_{r}=(2 \gamma-2 \alpha) r, \\
& p_{z}=(4 \alpha-4 \gamma) z,
\end{aligned}
$$

and therefore

$$
p=(\gamma-\alpha)\left(r^{2}-2 z^{2}\right) .
$$

The boundary conditions for the balance between stress and capillary-centrifugal forces are, in the case of a planar interface $z=h(r, t)=h_{\min }(t)$,

$$
\begin{aligned}
-p+2 \mu_{1} \frac{\partial u_{z}}{\partial z} & =-\frac{\Omega^{2}}{2} r^{2}, \\
\frac{\partial u_{z}}{\partial r}+\frac{\partial u_{r}}{\partial z} & =0
\end{aligned}
$$

so that, plugging in (30)-(32), we obtain

$$
\begin{aligned}
-(\gamma-\alpha)\left(r^{2}-2 h^{2}\right)+2\left(\alpha r^{2}-2 \gamma h^{2}\right) & =-\frac{\Omega^{2}}{2} r^{2}, \\
2 \alpha z r+2 \gamma r z & =0,
\end{aligned}
$$

resulting in

$$
\alpha=-\gamma=-\frac{\Omega^{2}}{8} .
$$

Since the center of the drop, at $r=0$, moves in the vertical direction, it will follow

$$
\frac{d h_{\min }}{d t}=u_{z}\left(r=0, h_{\min }\right)=\beta h_{\min }^{3}=-\frac{2}{3} \frac{\Omega^{2}}{8} h_{\min }^{3},
$$

Copyright $@$ by SIAM. Unauthorized reproduction of this article is prohibited. 
and we get

$$
h_{\min }(t) \sim \frac{\sqrt{6}}{\Omega} t^{-\frac{1}{2}}
$$

for $t \gg 1$.

Figure 8 represents the evolution of $h_{\min }^{-2}(t)$ and shows a comparison with a linear law for the last stages, confirming the asymptotic law (33). Figure 9 presents numerical profiles for $t \gg 1$ and the same profiles rescaled according to (29) in order to verify their convergence towards the similarity function $f(\xi)$.

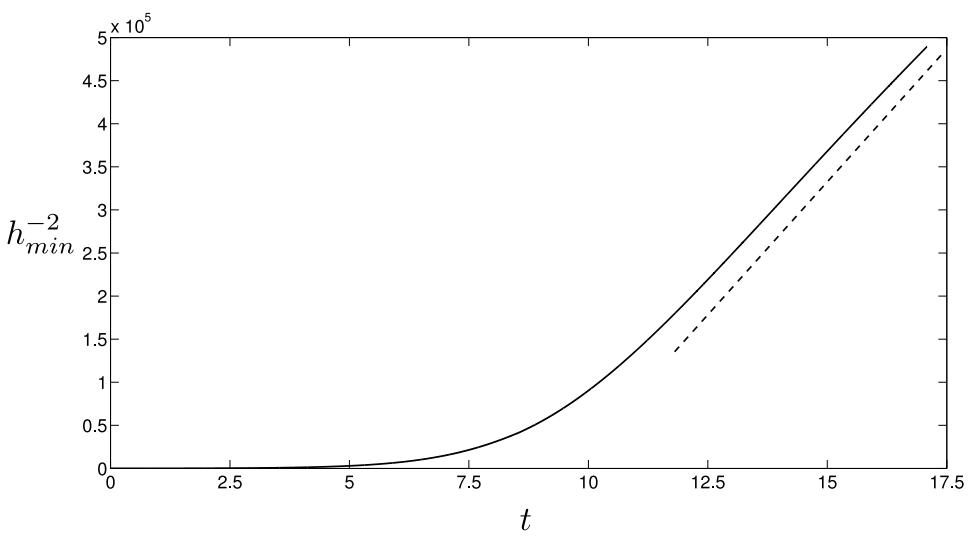

FIG. 8. Evolution of the inverse square of the film thickness at constant $L=2.54558$ and comparison with straight line for $t \gg 1$.

5. Evolution of drops in three dimensions. In the previous section we have discussed the evolution of axisymmetric rotating droplets with constant $\Omega$ and $L$. We paid special attention to the development of instabilities and the possibility of topological changes. Of course, a natural question arises concerning the stability of all these results under perturbations that break the axial symmetry. This requires analysis and numerical computations of the evolution in generic 3D situations.

5.1. Evolution at constant $\boldsymbol{\Omega}$. In the preceding section we showed that axisymmetric drops rotating at constant $\Omega$ for $\Omega>\Omega^{*}$ evolve into the so-called (in the notation introduced by Howell, Scheid, and Stone [15]) pizza shape. In the evolution, the film at the center tends to zero in finite time, as shown in [15]. Whether or not these axisymmetric shapes are stable or, on the contrary, evolve towards a different configuration was mentioned as an open problem. With our boundary elements code, we found that indeed the expanded pizza only keeps the axial symmetry when the angular velocity is sufficiently large, while for $\Omega<\Omega_{2} \in(3.28,3.31)^{1}$ drops tend to axisymmetric equilibrium spheroids. For $\Omega>\Omega_{2}$ and $\Omega<\Omega_{a p} \in(4.55,4.59)$ an initially spherical drop evolves into an unstable peanut that elongates infinitely in finite time. The drops become approximately axisymmetric about the $r$ axis, and one can develop very easily a thin jet model of the type described in [11], consisting of the following equations (in the simplified situation where we have only one fluid, although

\footnotetext{
${ }^{1}$ The value of $\Omega_{2}$ from [6] corresponds, in our units, to $\Omega_{2}=3.24$.
} 


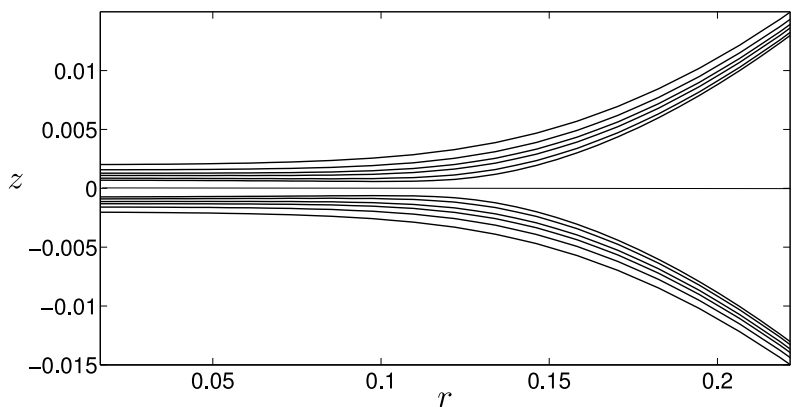

(a) Profiles without rescaling.

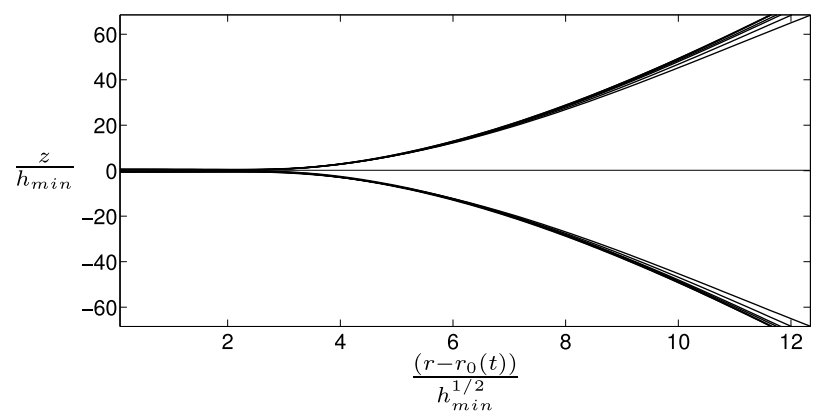

(b) Rescaled profiles.

Fig. 9. Profiles near the region where the thin film and the rim meet for $t \gg 1$. In (a) profiles are depicted without scaling, and in (b) the same profiles are represented after rescaling with the similarity law. Note that self-similarity behavior for constant $L>L^{*}$ shows convergence to toroidal type II solutions.

this can be easily generalized):

$$
\begin{aligned}
\frac{3 \mu}{h^{2}} \frac{\partial}{\partial r}\left(h^{2} \frac{\partial v}{\partial r}\right) & =\frac{\partial \Pi}{\partial r}, \\
\frac{\partial h}{\partial t}+\frac{h}{2} \frac{\partial v}{\partial r}+v \frac{\partial h}{\partial r} & =0,
\end{aligned}
$$

where

$$
\Pi(r, t)=\kappa(r, t)-\frac{\Omega^{2}}{2} r^{2} \simeq-\frac{\Omega^{2}}{2} r^{2}
$$

and $v(r, t)$ is the $u_{r}(r, z, t)$ component of the velocity field, which is, at leading order, independent of $z$ and dominant with respect to the $u_{z}$ component. Similarity solutions

$$
h(r, t)=\left(t_{0}-t\right)^{\alpha} f\left(\left(t_{0}-t\right)^{\beta} r\right), \quad v(r, t)=\left(t_{0}-t\right)^{\gamma} g\left(\left(t_{0}-t\right)^{\beta} r\right)
$$

to this system such that volume is preserved require $\alpha=\frac{1}{4}, \beta=\frac{1}{2}, \gamma=-\frac{3}{2}$. Notice that surface tension forces become negligible with respect to centrifugal forces for these similarity solutions. The equations for $f(\xi)$ and $g(\xi)$ are

$$
\begin{aligned}
\Omega^{2} \xi+\frac{3 \mu}{f^{2}} \frac{d}{d \xi}\left(f^{2} \frac{d g}{d \xi}\right) & =0, \\
f^{2}+\xi \frac{d}{d \xi}\left(f^{2}\right)-2 \frac{d}{d \xi}\left(f^{2} g\right) & =0
\end{aligned}
$$

Copyright (c) by SIAM. Unauthorized reproduction of this article is prohibited. 
and one straightforwardly computes the solutions to be of the form

$$
g(\xi)=\frac{\xi}{2}, \quad f(\xi)=C e^{-\frac{\Omega}{\sqrt{3 \mu}} \xi} .
$$

The constant $C$ must be chosen so that the drop has a volume $V$, yielding

$$
C=\sqrt{\frac{V \Omega}{\sqrt{3 \mu \pi^{3}}}} .
$$

Capillary forces enter into play only at the tips of the peanut and result in the formation of a small droplet whose size decreases in time. As in the axisymmetric case, one cannot expect infinite growth of the drop in finite time, since inertial terms in Navier-Stokes equations necessarily will be dominant before the drop spreads out to infinity. The result will be growth up to some sufficiently large length and the appearance of Rayleigh-Plateau instabilities, resulting in breakup of the elongated drop into smaller droplets.

If $\Omega>\Omega_{a p}$ and $\Omega<\Omega_{s p} \in(4.69,4.73)$, the initial spheroid configuration evolves towards a nonaxisymmetric pizza-like shape (a torus with a thin film in the middle) that is also unstable. Finally, for $\Omega>\Omega_{s p}$ the drop degenerates into an axisymmetric pizza that evolves as described in the previous section. We have depicted the four cases in Figure 10.
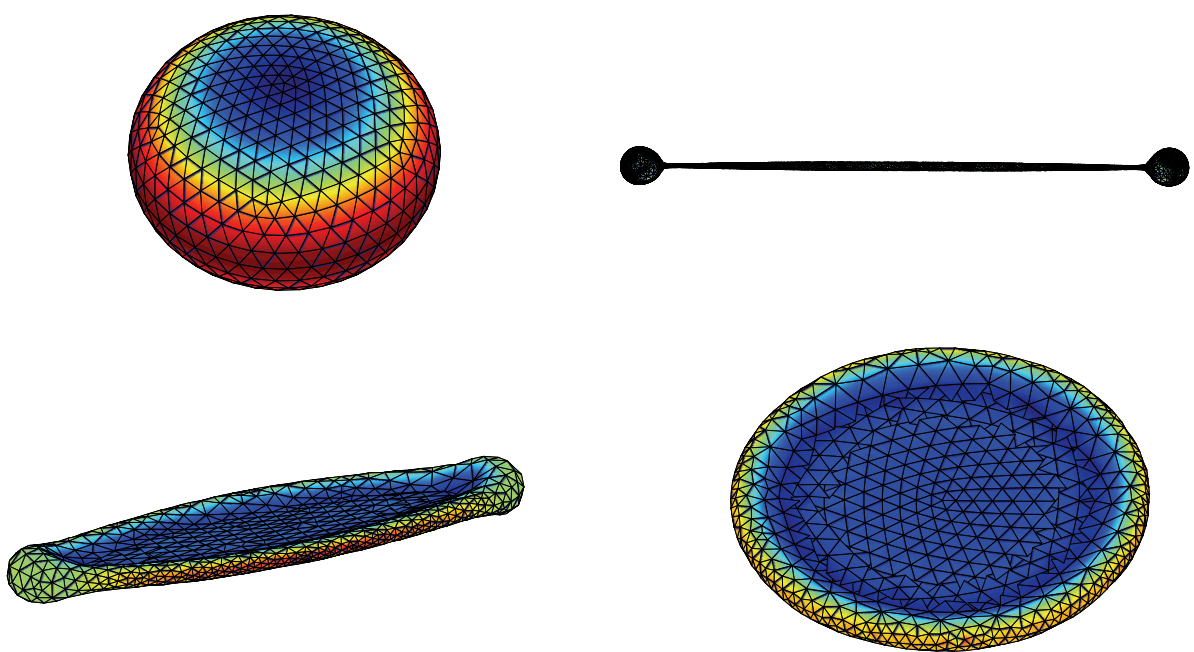

FIG. 10. Shapes resulting from the evolution of the rotating drop at constant $\Omega: \Omega<\Omega_{2}$ (top left), $\Omega_{2}<\Omega<\Omega_{a p}$ (top right), $\Omega_{a p}<\Omega<\Omega_{s p}$ (bottom left), and $\Omega>\Omega_{s p}$ (bottom right). They are not at the same scale. The values of $\Omega_{2}, \Omega_{a p}$, and $\Omega_{s p}$ are obtained numerically.

5.2. Evolution at constant $\boldsymbol{L}$. It is well known, since the works of Brown and Scriven [6], that bifurcations breaking axial symmetry may take place at various values of $L$. For $L=L_{2} \in(0.65,0.66)$, axially symmetric shapes become unstable, and evolution may lead to equilibrium shapes with a 2 -fold symmetry about the axis. We found that this is actually the case, and for $L>L_{2}$ but smaller than $L_{2}^{*} \in(1.06,1.10)$ evolution leads to the so-called peanut shapes. For $L>L_{2}^{*}$, interesting phenomena 


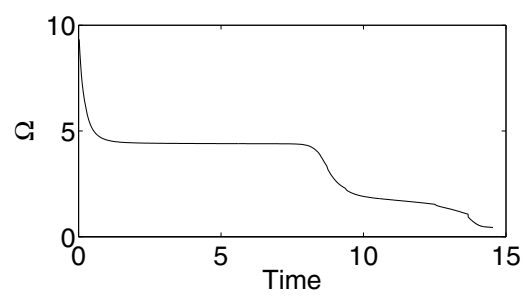

(a) $\Omega$ versus time for $L=1.41$.

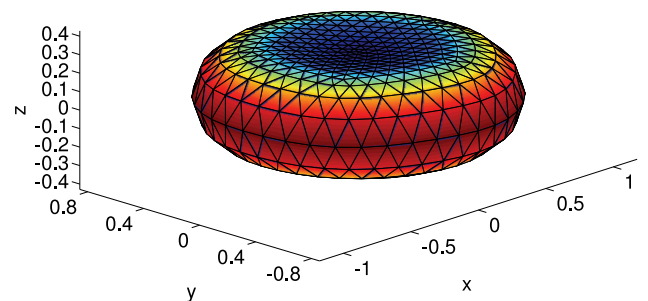

(b) $t=2.36$.

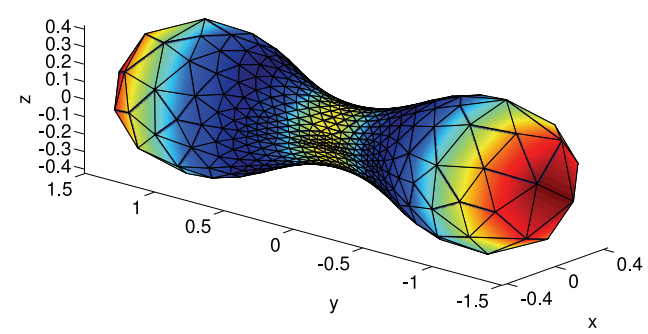

(d) $t=12.36$.

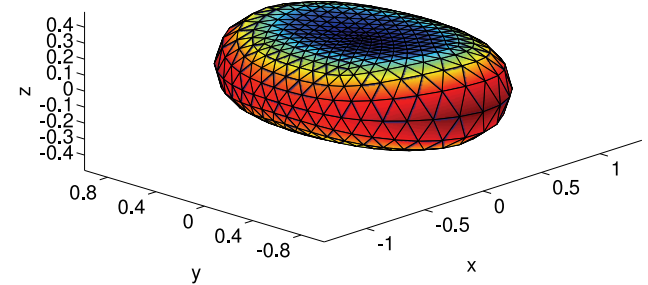

(c) $t=8.11$.

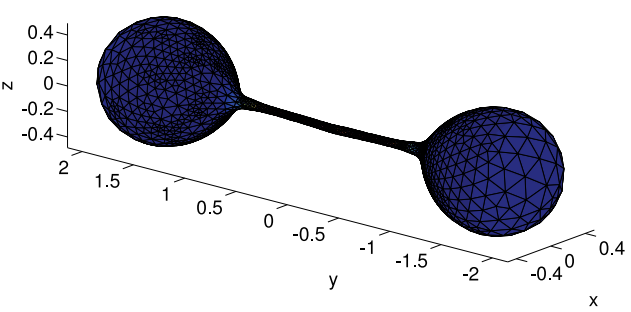

(e) $t=14.16$.

FIG. 11. Evolution of the rotating drop at constant $L$ for $L=1.41, \mu_{1}=1$, and $\mu_{2}=0.01$.

take place (see Figure 11). First, an initially spherical drop deforms into an axisymmetric shape. Nevertheless, these shapes are also unstable under nonaxisymmetric perturbations and after some time destabilize and evolve very quickly towards the peanut shape that is also unstable (since $L>L_{2}^{*}$ and there are not peanut-type equilibrium solutions), and breakup of the drop in several pieces takes place. It is worth noting that centrifugal forces near the breakup point are subdominant with respect to viscous and capillary forces, so that breakup occurs as theoretically described in [18] and [8]. With our code we were able to follow the evolution very close to the breakup point, and we could even see the formation of two generations of necks (see Figure 12).

There is another value of the angular momentum, $L_{3}^{*}$, that we estimated numerically as $L_{3}^{*} \in(1.74,1.78)$, so that for $L>L_{3}^{*}$ the dominant mode driving the symmetry-breaking instability is 3 -fold, and therefore evolution leads to the formation of three necks emerging from the center of the drop, ending with smaller drops 


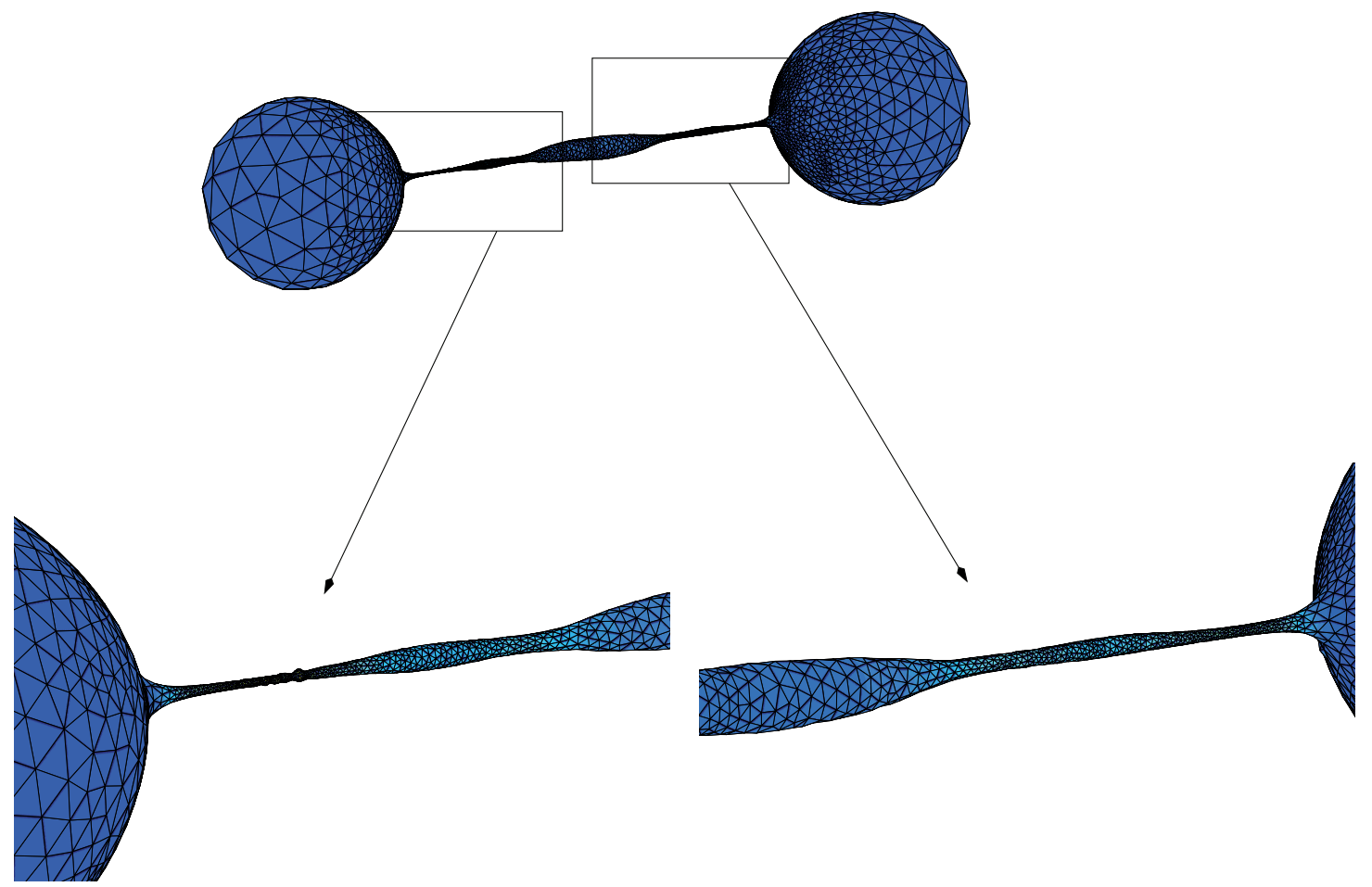

FIG. 12. Top: Shape of the rotating drop at constant $L$ near the breakup point $(t=14.55)$ for $L=1.41, \mu_{1}=1$, and $\mu_{2}=0.01$. The original drop has transformed into two big drops with a cascade of necks between them. Bottom: Detail of the necks. Observe the formation of small droplets in the smaller neck at the left.

TABLE 1

Axisymmetric evolution.

\begin{tabular}{|l|l|}
\hline Constant $\Omega$ & Constant $L$ \\
\hline \hline$\Omega<\Omega^{*}$ Axisymmetric equilibrium & $L<L^{*}$ Axisymmetric equilibrium \\
\hline$\Omega>\Omega^{*}$ Expanding pizza shape & $L>L^{*}$ Toroidal type II \\
\hline
\end{tabular}

and breaking up at a finite distance from the center (see Figure 13). We could not find solutions developing into an $n$-fold symmetry with $n>3$.

If we start with a torus as initial data, then for $L<L_{2}^{*}$ there is a clear tendency to close the hole so that the drop tends to an equilibrium shape with the same topology as the sphere. On the contrary, for $L>L_{2}^{*}$ the toroidal rim develops Rayleigh instabilities that lead to a breakup into a sequence of drops (see Figure 14).

6. Conclusions. In this article we have studied the evolution of rotating viscous drops. We have developed a numerical algorithm based on the boundary integral formulation of the Stokes system. The numerical algorithm is adaptive and automatically introduces local refinement in the critical regions such as necks, where the drop is going to break up. Based on the numerical results and the analysis of the equations, we have described the evolution both at constant angular velocity $\Omega$ and constant angular momentum $L$. The different regimes found are summarized in Tables 1 and 2 .

The values for $\Omega^{*}$ and $L^{*}$ have been determined numerically, $\Omega^{*}=4.3648 \ldots$, 


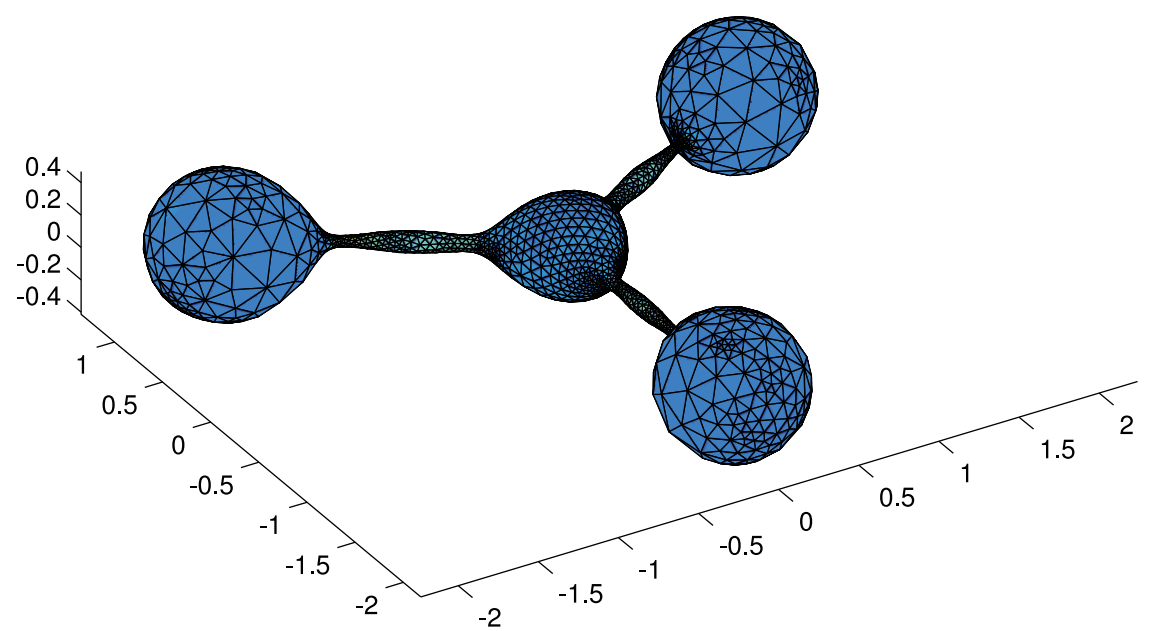

FIG. 13. Shape of the rotating drop at constant $L$ near the breakup point for $L=3.54$ and $\mu_{1}=\mu_{2}=0.5$. Observe that for $L>L_{3}^{*}$ the dominant mode driving the symmetry-breaking instability is 3 -fold.

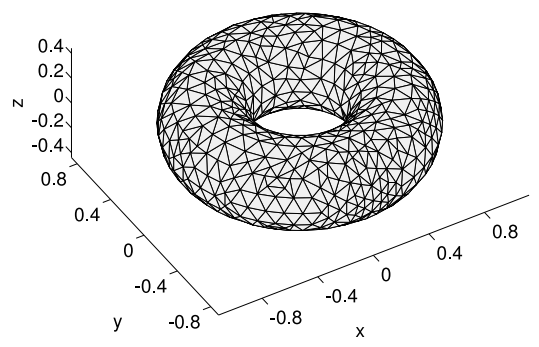

(a) Toroidal initial configuration.

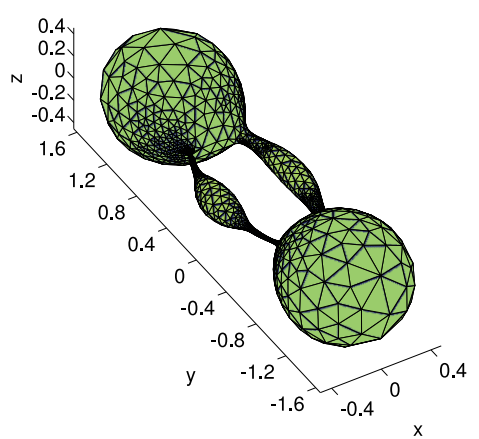

(c) Near the breakup point for $L=1.7$.

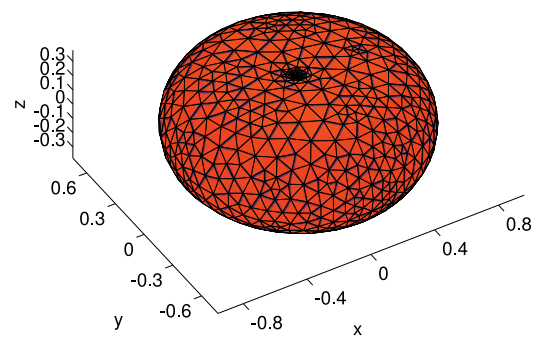

(b) Equilibrium shape for $L=0.85$.

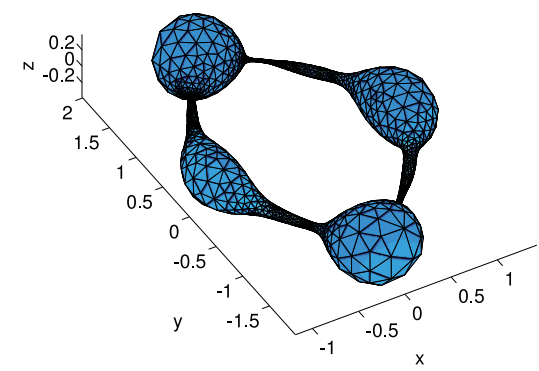

(d) Near the breakup point for $L=2.83$.

FIG. 14. Different equilibrium or breakup configurations starting from a torus as initial data. In all cases $\mu_{1}=\mu_{2}=0.5$.

$L^{*}=2.3755 \ldots$ Other critical values for $\Omega$ and $L\left(\Omega_{2}, \Omega_{a p}, \Omega_{s p}, L_{2}, L_{3}, L_{2}^{*}\right.$, and $\left.L_{3}^{*}\right)$ have been numerically computed for the particular case of a drop surrounded by a fluid 
TABLE 2

$3 D$ evolution.

\begin{tabular}{|l|l|}
\hline Constant $\Omega$ & Constant $L$ \\
\hline \hline$\Omega<\Omega_{2}$ Axisymmetric equilibrium & $L<L_{2}$ Axisymmetric equilibrium \\
\hline$\Omega_{2}<\Omega<\Omega_{a p}$ Elongating filament & $L_{2}<L<L_{2}^{*}$ Stable peanut shape \\
\hline$\Omega_{a p}<\Omega<\Omega_{s p}$ Asymmetric expanding pizza shape & $L_{2}^{*}<L<L_{3}^{*}$ Breakup (2-fold) \\
\hline$\Omega>\Omega_{s p}$ Axisymmetric expanding pizza shape & $L>L_{3}^{*}$ Breakup (3-fold) \\
\hline
\end{tabular}

with the same viscosity as the drop. We have verified that, for other viscosity ratios up to 0.01 , when the outer fluid viscosity is much smaller than the drop's viscosity, the qualitative picture does not change, although the critical values can be slightly different.

The approach, at constant $L>L^{*}$, to the toroidal type II solutions occurs at an $O\left(t^{-1 / 2}\right)$ rate, and the profiles for the interface present similarity features that we have described in detail. The elongating filaments at constant $\Omega$ reach an infinite length in finite time $t_{0}$, the length blows up at an $O\left(\left(t_{0}-t\right)^{-1 / 2}\right)$ rate, and the interface profile approaches an explicit self-similar solution. Breakup at constant $L$ is via an axisymmetric similarity profile of the type described by Lister and Stone [18] and Cohen et al. [8]. Finally, the axisymmetric expanding pizza shapes have a radius that grows at an $O\left(\left(t_{0}-t\right)^{-1 / 2}\right)$ rate, as shown by Howell, Scheid, and Stone [15].

A natural question that arises is whether the evolution for finite values of Ekman and Reynolds numbers presents different features from those of the limits considered in the present paper. This will be discussed in future publications.

Acknowledgments. The authors thankfully acknowledge the computer resources provided by the Centro de Supercomputación de Galicia (CESGA) and the cluster Odisea from the Universidad Autónoma de Madrid.

\section{REFERENCES}

[1] P. Ausillous And D. QuÉRÉ, Shapes of rolling liquid drops, J. Fluid Mech., 512 (2004), pp. 133-151.

[2] A. BEER, Einleitung in die mathematische Theorie der Elasticität und Capillarität, A. Gisen Verlag, Leipzig, 1869.

[3] S. I. Betelú, M. A. Fontelos, U. Kindelán, and O. Vantzos, Singularities on charged viscous droplets, Phys. Fluids, 18 (2006), 051706.

[4] N. Bohr And J. A. Wheeler, The mechanism of nuclear fission, Phys. Rev., 56 (1939), pp. $426-450$.

[5] M. Bonnet, Boundary Integral Equation Methods for Solids and Fluids, Wiley, New York, 1995.

[6] R. A. Brown and L. E. Scriven, The shape and stability of rotating liquid drops, Proc. R. Soc. Lond. A, 371 (1980), pp. 331-357.

[7] S. Chandrasekhar, The stability of a rotating liquid drop, Proc. R. Soc. Lond. A, 286 (1965), pp. $1-26$.

[8] I. Cohen, M. P. Brenner, J. Eggers, and S. R. Nagel, Two fluid drop snap-off problem: Experiments and theory, Phys. Rev. Lett., 83 (1999), pp. 1147-1150.

[9] V. Cristini, J. Blawzdziewicz, and M. Loewenberg, An adaptive mesh algorithm for evolving surfaces: Simulations of drop breakup and coalescence, J. Comput. Phys., 168 (2001), pp. 445-463.

[10] J. EgGers And M. A. Fontelos, The role of self-similarity in singularities of partial differential equations, Nonlinearity, 22 (2009), pp. R1-R44.

[11] J. Eggers and E. Villermaux, Physics of liquid jets, Rep. Prog. Phys., 71 (2008), 036601.

[12] M. A. Fontelos, U. Kindelán, And O. Vantzos, Evolution of neutral and charged droplets in an electric field, Phys. Fluids, 20 (2008), 092110. 
[13] R. GulLiver, Tori of prescribed mean curvature and the rotating drop, in Variational Methods for Equilibrium Problems of Fluids (Trento/Italy 1983), E. Gonzales and I. Tamanini, eds., Astérisque, 118 (1984), pp. 167-179.

[14] C. J. Heine, Computations of form and stability of rotating drops with finite elements, IMA J. Numer. Anal., 26 (2006), pp. 723-751.

[15] P. D. Howell, B. Scheid, And H. A. Stone, Newtonian pizza: Spinning a viscous sheet, J. Fluid Mech., 659 (2010), pp. 1-23.

[16] M. KallaY, Computing the moment of inertia of a solid defined by a triangle mesh, J. Graphics GPU \& Game Tools, 11 (2006), pp. 51-57.

[17] L. D. Landau and E. M. Lifshitz, Fluid Mechanics, Pergamon, Oxford, UK, 1984.

[18] J. R. Lister And H. A. Stone, Capillary breakup of a viscous thread surrounded by another viscous fluid, Phys. Fluids, 10 (1998), pp. 2758-2764.

[19] A. D. Myshkis, V. G. Babskit, N. D. Kopachevskit, L. A. Slobozhanin, and A. D. Tyuptsov, Low-Gravity Fluid Mechanics, Springer-Verlag, New York, Berlin, 1987.

[20] J. R. A. Pearson, Mechanics of Polymer Processing, Elsevier Applied Science Publishers, New York, 1985.

[21] J. Plateau, Mémoire sur les phénomènes que présente une masse liquide libre et soustraite à l'action de la pesanteur, Rev. Univ. Brux., 16 (1843), pp. 1-35.

[22] H. Poincaré, Sur l'équilibre d'une masse fluide animée d'un mouvement de rotation, Acta Math., 7 (1885), pp. 259-380.

[23] C. PozRIKIdis, Boundary Integral Methods for Linearized Viscous Flow, Cambridge Texts Appl. Math., Cambridge University Press, Cambridge, UK, 1992.

[24] H. M. Princen, I. Y. Z. Zia, And S. G. Mason, Measurement of interfacial tension from the shape of a rotating drop, J. Colloid Interface Sci., 23 (1967), pp. 99-107.

[25] J. M. Rallison And A. Acrivos, A numerical study of the deformation and burst of a viscous drop in an external flow, J. Fluid Mech., 89 (1978), pp. 191-200.

[26] D. R. Smith And J. E. Ross, Universal shapes and bifurcation for rotating incompressible fluid drops, Methods Appl. Anal., 1 (1994), pp. 210-228.

[27] O. Vantzos, Mathematical Modeling of Charged Liquid Droplets: Numerical Simulation and Stability Analysis, M.A. thesis, Department of Mathematics, University of North Texas, Denton, TX, 2006.

[28] T. G. Wang, A. V. Anilkumar, C. P. Lee, And K. C. Lin, Bifurcation of rotating liquid drops: Results from USML-1 experiments in space, J. Fluid Mech., 276 (1994), pp. 389-403.

[29] T. G. Wang, E. H. Trinh, A. P. Croonquist, and D. D. Ellemant, Shapes of rotating free drops: Spacelab experimental results, Phys. Rev. Lett., 56 (1986), pp. 452-455.

[30] A. Z. Zinchenko, M. A. Rother, And R. H. Davis, A novel boundary-integral algorithm for viscous interaction of deformable drops, Phys. Fluids, 9 (1997), pp. 1493-1511.

Copyright $@$ by SIAM. Unauthorized reproduction of this article is prohibited. 
Reproduced with permission of the copyright owner. Further reproduction prohibited without permission. 